DOI: $10.17957 / \mathrm{IJAB} / 15.1785$

http://www.fspublishers.org

\title{
A Protective dsRNA is Crucial for Optimum RNAi Gene Silencing in Chilo partellus
}

\author{
Olawale Samuel Adeyinka ${ }^{1,2^{*}}$, Idrees Ahmad Nasir ${ }^{1}$, Saman Riaz $^{1}$, Iqra Yousaf ${ }^{1}$, Nida Toufiq ${ }^{1}$, Abimbola Pius Okiki $^{2}$ \\ and Bushra Tabassum ${ }^{1,3}$ \\ ${ }^{1}$ Centre of Excellence in Molecular Biology, University of the Punjab, Lahore, Pakistan \\ ${ }^{2}$ Department of Biological Sciences, Afe Babalola University, Ado-Ekiti, Ekiti State, Nigeria \\ ${ }^{3}$ School of Biological Sciences, University of the Punjab, Lahore, Pakistan \\ *For correspondence: adeyinka.olawale@gmail.com \\ Received 02 January 2021; Accepted 25 March 2021; Published 10 May 2021
}

\begin{abstract}
RNAi technology is currently employed as an alternate control measure for agricultural pests. However, the variability of RNAi efficiency in insect pests limits the extensive usage of this technology and demands identifying the best target gene for effective RNAi. Four different bacterially-expressed dsRNA and purified dsRNAs coated on artificial diet were fed to the larvae. The transcripts expression was analyzed at 5 days and 15 days post-exposure to various dsRNAs. In the larvae fed on bacterially-expressed dsRNA, knockdown percentages were 80 and 57\% knockdown in Acetylcholinesterase transcript, 40 and $60 \%$ gene knockdown in Arginine kinase, 74 and $73 \%$ knockdown in Chymotrypsin, and 80 and $20 \%$ reduction in VATPase transcript expression. Overall, the mRNA knockdown percentages in the targeted genes were more pronounced at 5 days of exposure to bacterially-expressed crude dsRNA than 15 days of exposure. However, most purified dsRNAs rarely induce any significant knockdown except dsARG, which reduced the arginine kinase transcript by $40 \%$. Our findings suggest that for optimum RNAi in C. partellus, the dsRNA must be protected from direct access with nucleases. (C) 2021 Friends Science Publishers
\end{abstract}

Keywords: Agricultural pest; Bacterially-expressed dsRNA; C. partellus; Gene knockdown; RNAi technology

\section{Introduction}

Spotted stem borer attacks maize and causes significant yield losses (Mugo et al. 2002; Ajala et al. 2010). It is a substantial contributor to food insecurity in developing countries because millions of dollars are lost by smallholder farmers each year (Pratt et al. 2017). Climate change has a high tendency to accelerate its proliferation and increase the severity of its damages (Adeyinka et al. 2018) based on several prediction models (Tamiru et al. 2012; Khadioli et al. 2014). The rate of damage is still on the increase despite several control techniques currently available. The use of chemical control is mainly favored among several other measures. However, excessive use of pesticides can result in pest resurgence, an outbreak of secondary pests, and poses high risks to the environment and human health (Verger and Boobis 2013). One of the critical strategies to ensure a sustainable food supply is reducing crop losses due to insect pests through ecologically and economically integrated pest management (IPM) practices. Over the past decades, researchers have revealed the potential of RNAi technology to control insect pests.

The exogenous double-stranded RNA (dsRNA) induces degradation of mRNA sequences complementary to guide strand siRNA in organisms (Fire et al. 1998; Mello and Conte 2004; Ghosh et al. 2017; Worrall et al. 2019). RNAi pathway is initiated by RNase-III-like enzymes that cleave various dsRNAs into 20-25 nucleotide (nt) siRNA duplexes (Colmenares et al. 2007; Park et al. 2008;). The diced siRNAs generate guide strands and passenger strands with different thermodynamic properties based on asymmetry rule (Schwarz et al. 2003). The guide strands with the less stable 5' end favorably bind with Argonaute protein (Tim et al. 2004) to form a complex known as RNA-induced silencing complex (RISC). On loading to RISC, the targeted complementary regions of mRNA link with the guide strand and cleave the phosphodiester bond at the tenth and eleventh nucleotide from the guide strand 5' end (Elbashir 2001). The RNAi mechanism is currently applied in controlling Agricultural pests.

Recent advances in RNAi technology have drastically increased knowledge on the RNAi mechanism and gene knockdown in several insects (Lü et al. 2019; Vogel et al. 2019; Adeyinka et al. 2020; Husain et al. 2021; Jain et al. 2021). Scientists are currently improving the dsRNA delivery mode because the internalization of dsRNA is vital

To cite this paper: Adeyinka OS, IA Nasir, S Riaz, I Yousaf, N Toufiq, AP Okiki, B Tabassum (2021). A Protective dsRNA is Crucial for Optimum RNAi Gene Silencing in Chilo partellus. Intl J Agric Biol 25:1238-1248 
for effective RNAi in insects. RNAi silencing signals triggered by dsRNA(s) or siRNA(s) usually transport genetic regulatory information between cells via cellautonomous and non-cell autonomous. In the cellautonomous RNAi response, the silencing effect is limited to the cell, whereas in non-cell autonomous, the silencing effect is observed in all cells that can take up the dsRNA (environmental RNAi), and the silencing signal is transported from the specific cell to other cells or tissues (Systemic RNAi). The systemic RNA interferencedefective-1 (SID-1) protein and endocytosis are currently the best-studied dsRNA uptake mechanisms. Several SID mutants (SID 1, SID2, SID 3, and SID 5) have been studied in $C$. elegans to expand scientific knowledge on systemic RNAi pathways and SID-1 orthologues have been reported in insects. However, the presence of sid-1-like genes does not necessarily result in a robust systemic RNAi response (Miyata et al. 2014) in the insect. The endocytosis pathway is the popular dsRNA uptake mechanism in most insects. Clathrin-mediated endocytosis has been investigated as the dsRNA-uptake route in insect species (Li et al. 2015; Cappelle et al. 2016; Pinheiro et al. 2018; Abbasi et al. 2020). The efficacy of RNAi in an insect is generally influenced by dsRNA uptake. Several factors, such as physiological $\mathrm{pH}$, targeted genes, and nucleases, influence insects' RNAi efficiency (Dias et al. 2020; Zhu and Palli 2020). Identifying the effective targets for specific insect pests is a major challenge in RNAi-mediated biopesticide. This study aims to identify the best target gene and the best dsRNA form that can be used for optimum RNAi silencing of $C$. partellus as potential control measure.

We examined four dsRNA stability and their ability to initiate the RNAi pathway in $C$. partellus. Bacterially expressed dsRNA and purified dsRNA of the genes (VATPases, Acetylcholinesterase, Chymotrypsin, Arginine kinase) that have been explored in Lepidoptera were fed to C. partellus. The knockdown efficiency was evaluated at different time intervals. The data presented in this study establishes that oral feeding of bacterially expressed dsRNA induced significant RNAi knockdown effects.

\section{Materials and Methods}

\section{Insect management}

Maize stem borers ( $C$. partellus) larvae were reared at our insectary facility in the Centre of Excellence in Molecular Biology, Pakistan. To adapt to the artificial new rearing environment $\left(26 \pm 2^{\circ} \mathrm{C}\right.$ under a $14: 10 \mathrm{~h}$ light: dark and $65 \pm$ $5 \%$ relative humidity), the larvae were maintained for 2 days on fresh maize stem before given an artificial diet.

\section{Target selection and primer design}

Several essential genes necessary for insects' survival, especially in Lepidoptera, were mined from literature. The genes were selected across functions like digestive enzymes (Chymotrypsin), cellular energy metabolism (Arginine kinase), central nervous system (Acetylcholinesterase) and ATP hydrolysis to transport protons across intracellular and plasma membranes (Vacoular-ATPase). C. partellus lacks adequate nucleotides information for the selected genes, so primers were designed for consensus sequences from available lepidopteran homologs in NCBI databases. We selected nucleotide information of 7 different insect limited to the Pyraloidea subfamily of Lepidoptera (Chilo suppressalis, Helicoverpa armigera, Bombyx mori, Spodoptera litura, Plutella xylostella, Scirpophaga incertulas, Amyelois transitella). The FASTA format sequences were imported into Ugene software and aligned with Clustal omega add-in to obtain the consensus sequence. Gene-specific primers (Table 1) were designed with HindIII and Xbal restriction enzymes using online primer 3 plus software (www.bioinformatics.nl/primer3plus).

\section{RNA Isolation and cDNA preparation}

Total RNA was extracted from a pool of five larvae comprised of different developmental stages using TRI Reagent (Sigma-Aldrich, St. Louis, U.S.A.) according to the manufacturer instructions. DNaseI was used to remove DNA impurities and RNA's concentration and purity was determined by A Nanodrop ND-1000 spectrophotometer. The RNA's integrity was verified on $1.5 \%$ TAE gel. $1 \mu \mathrm{g}$ of the RNA was used to synthesize cDNA with Oligo dT primers, using RevertAid First Strand cDNA synthesis kit according to the manual instruction.

\section{Targeted gene sequences identification}

To identify the targeted gene sequences, PCR amplification was performed in GeneAmp PCR system 9700 Thermal Cycler (Applied Biosystems, Singapore). The reaction mixture $(20 \mu \mathrm{L})$ contained $2 \mu \mathrm{L}$ of $10 \mathrm{X}$ Tag Buffer, 0.4 $\mathrm{mM}$ of $\mathrm{MgCl}_{2}, 0.15 \mathrm{~m} M$ of dNTP $1.25 \mathrm{U}$ of Taq, $0.5 \mu M$ of both forward and reversed primers, and $1 \mu \mathrm{g}$ of cDNA. The amplification was performed according to the following profile: initiation at $95^{\circ} \mathrm{C}$ for $5 \mathrm{~min}$, followed by 35 cycles of $95^{\circ} \mathrm{C}$ for $30 \mathrm{~s}, 58^{\circ} \mathrm{C}$ for $30 \mathrm{~s}$, and $72^{\circ} \mathrm{C}$ for $30 \mathrm{~s}$. The amplified products were ligated in pCR2.1 vector and transformed into $E$. coli top 10 competent cells. Positive clones were confirmed through restriction digestion and sequencing.

\section{Bioinformatics analysis}

The revealed sequences of four genes used in this study were compared to known the sequences in NCBI database using the NCBI BLAST server (http://www.ncbi.nlm.gov/BLAST). The molecular weights and theoretical pIs were predicted using the Compute $\mathrm{pI} / \mathrm{Mw}$ tool (https://web.expasy.org/compute_pi/). The 
sequences were aligned by muscle algorithm and used to construct phylogenetic tree analysis based on the neighborjoining method using MEGA X with 1,000 Bootstrap values.

\section{Construction of dsRNA expression vector and induction}

Each of the four individual genes was digested with Hind III and Xbal restriction enzymes and ligated to L4440 vector (Fig. 1). We first transformed and confirmed the transformant in E. coli top 10 followed by transformation into E. coli HT115 strain. Positive E. coli HT115 colonies were grown overnight in $5 \mathrm{~mL}$ YT media (Yeast extract 10 $\mathrm{g} / \mathrm{L}$, Bacto peptone $5 \mathrm{~g} / \mathrm{L}, \mathrm{NaCl} 10 \mathrm{~g} / \mathrm{L}$ ) containing $100 \mu \mathrm{g}$ $\mathrm{mL}-1$ ampicillin at $37^{\circ} \mathrm{C}$. The cultures were diluted to 500 $\mathrm{mL}$ and incubated until it reaches $\mathrm{OD}_{595}=0.4$. Then, the dsRNA synthesis was induced by the addition of $0.6 \mathrm{mmol}$ 1-1 IPTG at $37^{\circ} \mathrm{C}$ for another $3-4 \mathrm{~h}$. Raw bacteriallyexpressed dsRNAs were harvested and some of the harvested cells were purified as we described in our initial publication (Adeyinka et al. 2019).

\section{Fitness of dsRNA at insectary condition and on artificial diet}

We investigated if the condition at the insectary would have any effect on dsRNA stability. For this, dsRNA was diluted with nuclease-free water and exposed in the growth chamber for various time intervals: $0 \mathrm{~h}, 24 \mathrm{~h}, 48 \mathrm{~h}$, and $72 \mathrm{~h}$. The samples were later resolved on $1 \%$ agarose gel electrophoresis to evaluate the effect of environmental conditions. Furthermore, the dsRNA was overlaid on the artificial diet for $36 \mathrm{~h}$ and $72 \mathrm{~h}$ to assess the time taken for dsRNA degradation. The dsRNA was isolated from the treated diets by Tri-reagent and resolved on $1 \%$ gel to estimate the effect of diet on dsRNA stability.

\section{In vitro stability of dsRNA in Insect heamolmyph}

To extracted haemolymph, an incision was made at the larvae's prolegs with a surgical blade, and the haemolymph was gradually extracted with a $10 \mu \mathrm{L}$ pipette. Furthermore, $1 \mu \mathrm{g}$ of dsRNA was used to incubate $2 \mu \mathrm{L}$ of the haemolymph at various time intervals to reveal their stability.

\section{Gut content stability test}

C. partellus larvae were dissected to extract the entire gut content. Briefly, a surgical blade was used to make minor incisions around the anus and the larvae's neck region; forceps were used to pull out the whole gut into $1 \mathrm{X}$ PBS in a 0.2 $\mathrm{mL}$ tube. The whole gut was inserted into a $500 \mu \mathrm{L}$ Eppendorf tube with a slight cut and then centrifuged at centrifugation at $13000 \times g$ for $15 \mathrm{~min}$ at $4^{\circ} \mathrm{C}$ into a 1.5 $\mathrm{mL}$ Eppendorf tube containing ice-cold $1 \times$ phosphatebuffered saline (PBS, pH 7.4) with $1 \mathrm{mg}$ phenylthiourea
(PTU). $1 \mu \mathrm{g}$ of dsRNA was mixed with $2 \mu \mathrm{L}$ of the haemolymph and incubated for various time intervals to evaluate its stability. The dsRNA integrity was examined on $1 \%(\mathrm{w} / \mathrm{v})$ agarose gels.

\section{Temporal expression of the four targeted genes}

To estimate the targeted genes relative transcription levels, samples were collected across developmental stages from three technical replicates and two independent biological replicates. $1^{\text {st }}$ instar larvae were used as reference samples for the temporal expression profiling analyses. RNA isolation and synthesize cDNA methods were the same described above. Beta-tubulin was used as an internal control for normalization of transcript abundance across developmental stages based on our initial validation report (Adeyinka et al. 2019). RT-qPCR was conducted using SYBR premix with the primers shown in Table 1. Data were analyzed by the $2-\Delta \Delta C T$ method, ANOVA was used to analyzed expression, and the means were separated using Fisher's protected Least Significant Difference (LSD) test for significance using GraphPad Prism 7 software

\section{Insect bioassay to evaluate the effect of various dsRNAs used in this study}

To compare the knockdown efficiency in bacterially expressed dsRNA and purified dsRNA of various targeted genes, $2^{\text {nd }}$ instar larvae were starved for about $1 \mathrm{~h}$ before exposure to the dsRNA treatments. For the crude bacteria feeding experiment, $100 \mu \mathrm{L}$ of dsRNA inducing bacteria was overlaid on artificial diet and fed to $2^{\text {nd }}$ instar larva for 21 days. All diets were replaced after $24 \mathrm{~h}$. In a parallel experiment, $20 \mu \mathrm{g}$ of purified dsRNA was overlaid on the artificial diet. A total of 20 larvae were used in each treatment in triplicate, and an empty L4440 vector was used as the control treatment. After 5 days and 15 days of exposure to dsRNA, two larvae per replicate were collected and ground in Trizol for RNA isolation and subsequent RT-qPCR.

\section{Transcript expression by RT-qPCR and statistical analysis}

Real-time PCR was performed to evaluate the knockdown in mRNA expression of all four targeted genes after dsRNA exposure. The synthesized cDNA was diluted with nuclease-free water in 1:10 ratio and used for RT-qPCR. The RT-qPCR assays were conducted according to Minimum Information for Publication of Quantitative RealTime PCR Experiments guidelines. RT-qPCR was performed in triplicate in a Piko-real 96 Realtime PCR system (Thermo scientific) to evaluate knockdown brought about by each specific dsRNA. ELF gene expression was used as an internal reference to normalized the data based on our validation findings (Adeyinka et al. 2019). 
Table 1: List of primers used in the study with specific amplicons size

\begin{tabular}{ll}
\hline Gene name & PCR Primers $\left(5^{\prime}-3^{\prime}\right)$ \\
\hline Acetylcholinesterase & F:AAGCTTTGCCTTCTTCATCATCGTG \\
& R:TCTAGATGGGATCAACAGTTGGCTATC \\
Arginine kinase & F:AAGCTTTTGACTGGGCCTCTGTGA \\
& R:TCTAGACGCAACCCTTGAGAAATTG \\
Vacuolar ATPase & F:AAGCTTTCACGGAAGTACTCGGATAGAG \\
& R:TCTAGAACGGCGAGAAGGAGAAGTA \\
Chymotrypsin & F:AAGCTTTGTGCAAATGTTGGAGTCCT \\
& R:TCTAGAGTCCACCTCGAGGATTCTATTG \\
Acetylcholinesterase & RT-qPCR Primers (5'-3') \\
Arginine kinase & F:TGCCTTCTTCATCATCGTG R:GACTGCATGCGTGGAGTAGA \\
Chymotrypsin & F:ATTCCAACACCAGAGTCCAAGT R:AAGTCGCTGCTGAAGAAGTACC \\
Vacuolar ATPase & F:CAGCTACCGTGCATAACAACAT R:GTACTGACCGCTGCTCACTG \\
\hline
\end{tabular}
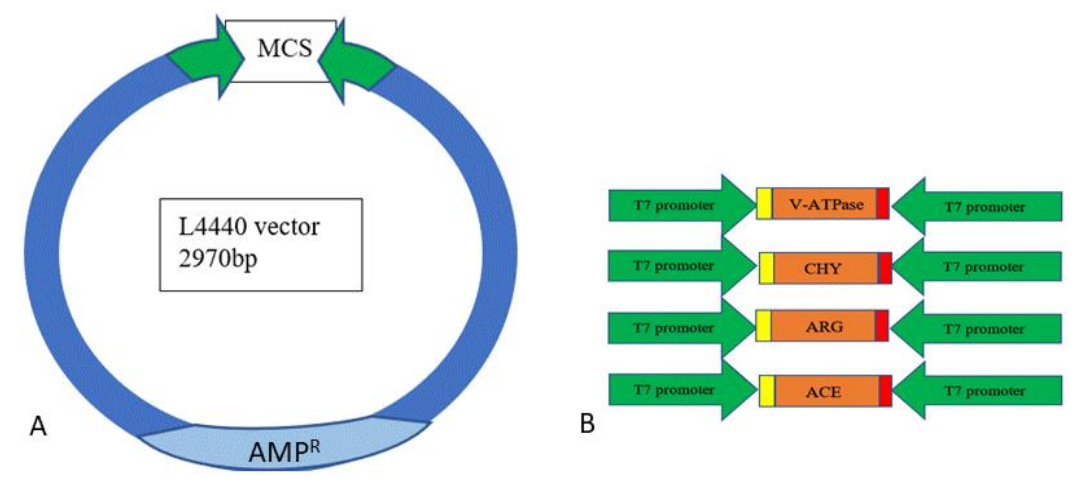

Fig. 1: Schematic representation of L4440 construct transformed in E. coli HT115 host. A) Demonstrating the L4440 vector containing multiple cloning site (MCS) with double T7 promoters (green colour), B) indicating the various gene that was cloned into the HindIII (yellow colour) and Xbal (red colour) restriction enzymes sites

Livak method (Livak and Schmittgen 2001) was used to determine the extent of gene expression. The amplifications cycling profile used comprise of an initial denaturation at $95^{\circ} \mathrm{C}$ for $5 \mathrm{~min}, 35$ cycles of denaturation at $95^{\circ} \mathrm{C}$ for 30 $\mathrm{s}$, annealing at $58^{\circ} \mathrm{C}$ for $30 \mathrm{~s}$, and extension at $72^{\circ} \mathrm{C}$ for $30 \mathrm{~s}$. The reaction mixture comprises of nuclease free water, cDNA Maxima SYBR Green qPCR 2X Master mix, and $500 \mathrm{n} M$ of primers. Analysis of variance was conducted to analyse the mean transcript expression of various dsRNA treatments to nuclease-free water fed larvae. Pairwise comparisons between treatments were performed with the Turkey test at $P<0.05$.

\section{Results}

\section{Amplification of targeted genes}

Several crucial genes necessary for the survival of insects have been effectively silenced in the insect. Among these crucial genes were Vacuolar-ATPase, Arginine kinase (Camargo et al. 2016), Acetylcholinesterase (Ye et al. 2017), and Chymotrypsin previously reported as potential target for RNAi mediated pest control. These four essential insect genes were amplified from $C$. partellus using specific primers (Table 1). The PCR products resolved on $1 \%$ agarose gel revealed amplification of a single fragment indicating the high specificity of individual genes (Fig. 2).

\section{Sequence comparison and phylogenetic analysis}

To examine the relatedness of the sequenced genes, the individual sequence was analyzed through BLASTn. The outcome indicated high sequence homology with other lepidopteran insects available in NCBI database. Specifically, the Chymotrypsin gene sequence showed $98 \%$ similarity with Chymotrypsin gene of Helicoverpa armigera; Vacuolar ATPase was $87 \%$ similar to $M$. sexta vacuolar ATPase; Arginine kinase gives $87 \%$ similarity with Spodoptera litura Arginine kinase and Acetylcholinesterase showed $91 \%$ identity to Chilo auricilius Acetylcholinesterase. The Table 2, highlights some of the chemical properties of the genes used in this study, their molecular weight, isoelectric point and number of amino acids. The BLASTn result were aligned by ClustalW in MEGA-X software while neighbour-joining method was used for the construction of phylogeny tree for individual gene to demonstrate their relatedness among the lepidopteran (Fig. 3). The phylogeny tree indicated that these genetic sequences are highly conserved among Lepidoptera. 
Table 2: Chemical properties of the targeted genes revealed through in-silico studies

\begin{tabular}{llll}
\hline Gene name & Accession no. & Isoelectric point & Molecular weight \\
\hline Acetylcholinesterase & MK560447 & 4.70 & 15652.83 \\
Arginine kinase & MK560449 & 5.02 & 15491.43 \\
Vacuolar ATPase & MK560450 & 6.24 & 26496.39 \\
Chymotrypsin & MK560452 & 8.09 & 7793.83 \\
\hline
\end{tabular}
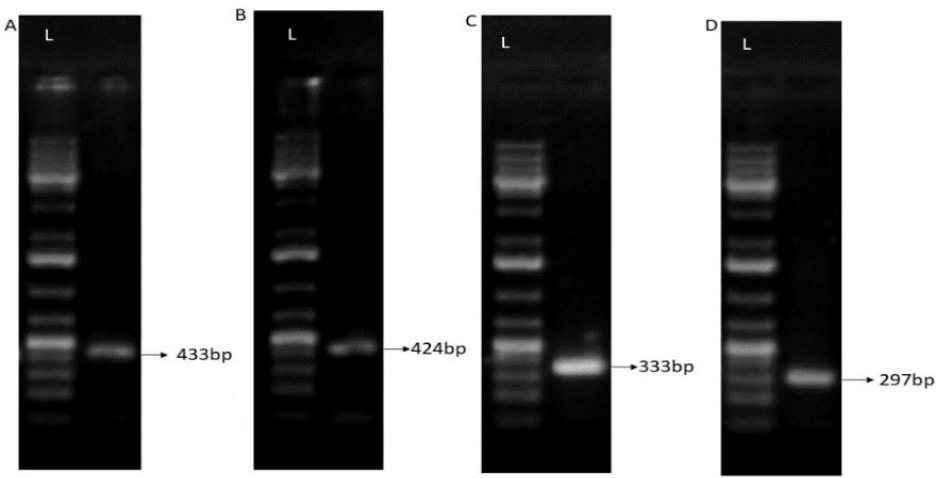

Fig. 2: PCR amplification of genes used in this study from C. partellus. A) Acetylcholinesterase gene fragment, B) Arginine kinase gene fragment, C) Chymotrypsin gene fragment, and D) Vacuolar ATPase gene fragment. L refers to $1 \mathrm{~kb}$ plus DNA ladder. The gene sequences were submitted in NCBI GenBank and assigned with the following accession numbers; Acetylcholinesterase - MK560447, Arginine kinase - MK560449, Vacuolar ATPase - MK560450, and Chymotrypsin - MK560452



Fig. 3: Phylogenetic tree analysis showing the relationship among C. partellus amplified genes and related sequences available in NCBI repository. A) Acetylcholinesterase, B) Chymotrypsin, C) Arginine kinase and D) V-ATPase genes. The analysis was based on the neighbour-joining method according to amino-acid sequences using MEGA-X. with 1,000 Bootstrap values

\section{E. coli HT115 induces dsRNA synthesis upon IPTG induction}

The individual constructs harboring each of the four amplified genes cloned in the L4440 vector were transformed into E. coli HT115 strain. The clones were confirmed through restriction digestion, as depicted in Fig. 4. Confirmed clones with two distinctly restricted fragments, one of $\sim 2.7 \mathrm{~kb}$ corresponding to the L4440 vector, while the second $\sim 500 \mathrm{bp}$ corresponding to transgene (Fig. 4) were selected for subsequent induction.
The recombinant HT115 strain transcribes high quantity dsRNA when induced with 0.6 mM IPTG (Fig. 5). A clear difference was observed between un-induced and induced cultures in terms of dsRNA production. The sizes of the transcribed dsRNAs correspond to individually targeted dsRNA in $C$. partellus.

\section{Stability test for purified dsRNAs}

The stability of the purified dsRNA was evaluated under various conditions before the bioassay analysis. 


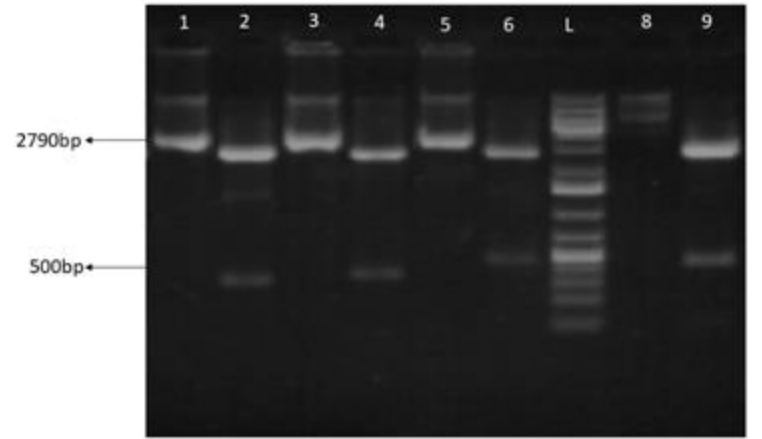

Fig. 4: Restriction digestion of positive clones confirming the specific gene insertion in the L4440 vector. Lane 1; undigested Vacuolar ATPase, Lane 2; digested Vacuolar ATPase, Lane 3; undigested Chymotrypsin, Lane 4; digested Chymotrypsin, Lane 5; undigested plasmid DNA with Acetylcholinesterase gene cloned, Lane 6; digested plasmid DNA harboring Acetylcholinesterase gene, L is the $1 \mathrm{kp}$ plus Ladder, Lane 8; undigested Arginine kinase, Lane 9; digested Arginine kinase

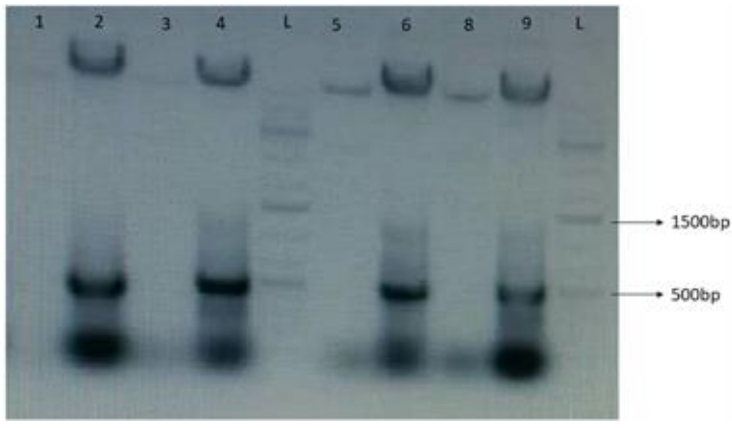

Fig. 5: Synthesis of double-stranded RNAs from IPTG-induced HT115 bacterial culture. Lane 1; un-induced Acetylcholinesterase, Lane 2; induced Acetylcholinesterase, Lane 3; un-induced Arginine kinase, Lane 4; induced Arginine kinase, Lane 5; un-induced Vacuolar ATPase, Lane 6; induced Vacuolar ATPase, Lane 6; induced Vacuolar ATPase, Lane 7; un-induced Chymotrypsin, Lane 8; induced Chymotrypsin. L is the $1 \mathrm{kp}$ plus Ladder

There was no significant reduction in the dsRNA quantity after $24 \mathrm{~h}, 48 \mathrm{~h}$, and $72 \mathrm{~h}$ after dsRNAs exposure to general insectary lab conditions. The same level of dsRNA intensity was observed within $24 \mathrm{~h}$ to $72 \mathrm{~h}$ of exposure (Fig. 6). These results indicated that the insectary lab conditions do not have any effect on dsRNA quality. Furthermore, we evaluated the stability of dsRNA overlaid on an artificial diet. A significantly high intact dsRNA was recovered after $36 \mathrm{~h}$ of exposure to an artificial diet (Fig. 7A). However, after $72 \mathrm{~h}$ of exposure on diet, almost the whole intact dsRNA was degraded (Fig. 7B). Based on these findings, artificial diet was replaced with a new diet overlaid with fresh dsRNA within every $24-30 \mathrm{~h}$ in subsequent feeding assays.

To determine dsRNA's fate inside $C$. partellus, the haemolymph was collected and incubated with dsRNA for various time intervals. The dsRNA was stable for $1 \mathrm{~min}$ and the stability prolonged for $5 \mathrm{~min}$; however, it gradually

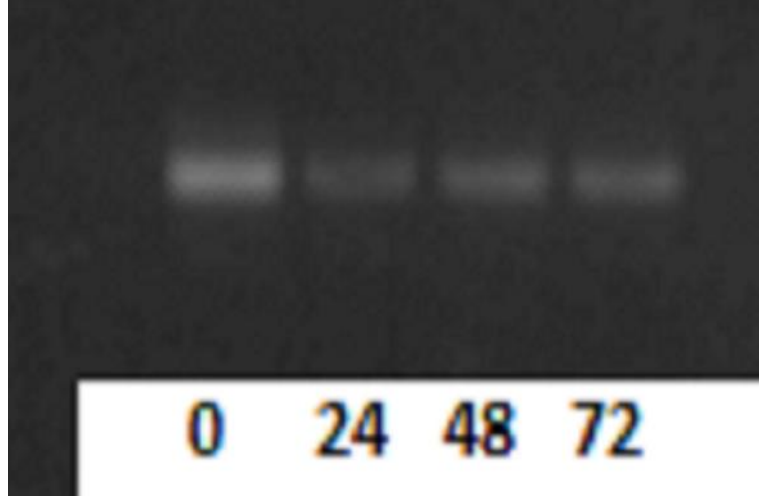

Fig. 6: dsRNA stability test in lab environment. dsRNAs were exposed to lab environment for variable periods $(0 \mathrm{~h}, 24 \mathrm{~h}, 48 \mathrm{~h}$, $72 \mathrm{~h}$ ), and their integrity was evaluated through agarose gel electrophoresis

degraded as the time interval increased (Fig. 8A). Surprisingly, the dsRNA degraded very fast in about 30 seconds when it was incubated with whole gut content for the time intervals; 30sec, $1 \mathrm{~min}, 2 \mathrm{~min}, 3 \mathrm{~min}, 4 \mathrm{~min}$ and 5 min (Fig. 8B). These results depict that gut contents have a high concentration of dsRNase (s). Based on these findings, we opted for a high dsRNA concentration in subsequent feeding assay to initiate effective RNAi.

\section{Temporal expression of Acetylcholinesterase, arginine kinase, $V$-ATPase, and Chymotrypsin}

The expression of the four genes was determined across all the developmental stages. Our data indicated that the analysis of the temporal expression of the targeted four genes: Acetylcholinesterase, arginine kinase V-ATPase, and Chymotrypsin were expressed in all the developmental stages with minimal expression in $3^{\text {rd }}$ and $4^{\text {th }}$ instar (Fig. 9).

\section{Bacterially expressed crude dsRNAs exhibit more effective knockdown compared to naked dsRNA}

To compare the knockdown sensitivity in larvae fed on purified dsRNA and crude bacterially-expressed dsRNA treatment at 5 and 15 days of exposure. The RT-qPCR analysis indicated Acetylcholinesterase was downregulated by up to 80 and $57 \%$ in the larvae fed on bacteriallyexpressed dsACE at 5 and 15 days exposure, respectively. Whereas larvae fed on purified dsACE did not show any significant knockdown at the same time of exposure (Fig. $10 \mathrm{~A}$ and $11 \mathrm{~A}$ ). The larvae fed with bacterially expressed dsARG exhibited 40 and $60 \%$ knockdown in the Arginine kinase transcript expression after 5 and 15 days of exposure, respectively (Fig. 10B). A similar knockdown level ( 40\%) in Arginine kinase was observed in larvae fed on purified dsARG (Fig. 11B). Fig. 11C showed that mRNA abundance of Chymotrypsin decreased by 74 and $73 \%$ at 5 and 15 days post-exposure to bacterially-expressed dsRNA, respectively. 




Fig. 7A: dsRNA stability test recovered from artificial diet after 36 hours of exposure. Lane 1: dsV-ATPase, Lane 2: dsACE, Lane 3: dsCHY, Lane 4: dsARG, while C refers to control diet overlaid with nuclease-free water

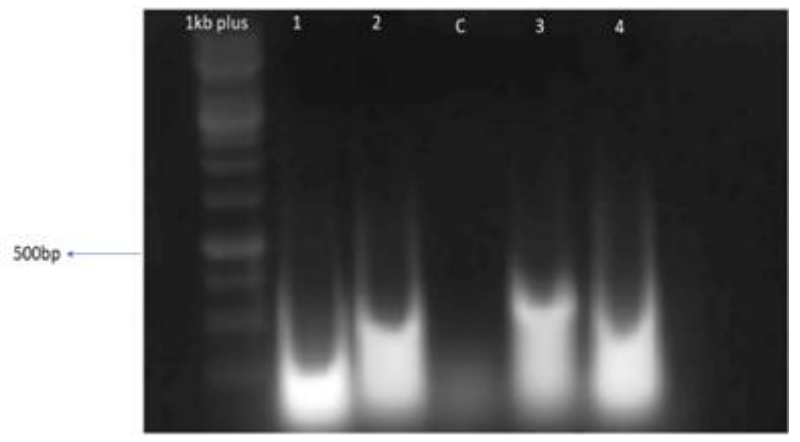

Fig. 7B: dsRNA stability test recovered from artificial diet after 72 hours of exposure. Lane 1: dsV-ATPase, Lane 2: dsACE, Lane 3: dsCHY, Lane 4: dsARG, while C refers to control diet overlaid with nuclease-free water

In contrast, purified dsCHY failed to exhibit any knockdown in the mRNA expression of the chymotrypsin gene in larvae fed with purified dsCHY (Fig. 11C). VATPase mRNA expression was reduced to about $80 \%$ when fed with bacterially-expressed V-ATPase dsRNA for 5 days, while extended exposure to 15 days resulted in $20 \%$ reduction in the transcript expression (Fig. 10D). Overall, the knockdown percentages for all targeted genes were more pronounced and significant at 5 days of exposure to bacterially-expressed dsRNA as compared to 15 days of exposure. On the other hand, purified dsRNAs rarely induced any significant knockdown percentage for all targeted genes except dsARG (Fig. 11B).

\section{Discussion}

The knockdown of essential genes in agricultural pests has been investigated as an alternative technique to control insect pests. However, some lepidopterans are refractory to RNA interference, and silencing has not been effective. Identifying relevant target genes for RNAi in insects is one of the hurdles that must be solved for effective RNAi-



Fig. 8A: Degradation of dsARG incubated with haemolymph derived from $C$. partellus for different time intervals. $\mathrm{L}$ refers to the $1 \mathrm{~kb}$ plus Ladder, and $\mathrm{C}$ refers to control (dsRNA without haemolymph). dsRNA incubated with haemolymph for; $1 \mathrm{~min}$ (lane 1), 5 min (lane 2), $10 \mathrm{~min}$ (lane 3), $15 \mathrm{~min}$ ((lane 4)), $30 \mathrm{~min}$ (lane 5), $60 \mathrm{~min}$ (lane 6) respectively

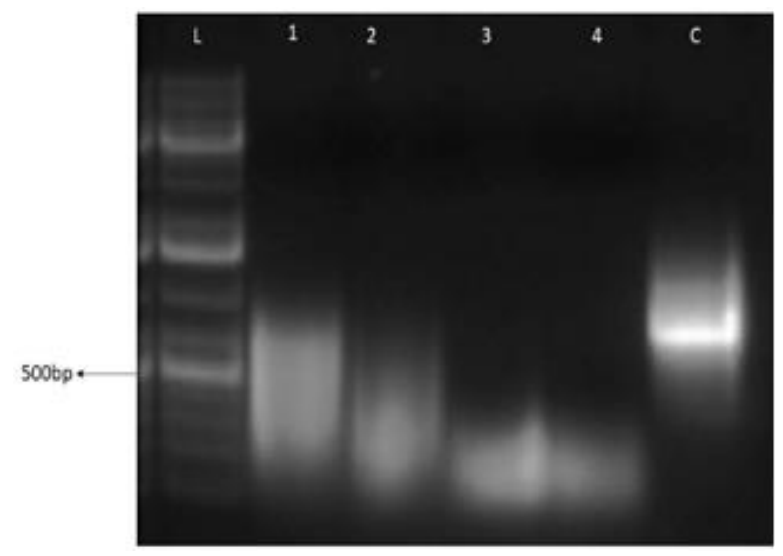

Fig. 8B: Degradation of dsARG incubated with gut contents derived from C. partellus for different time intervals. $\mathrm{L}$ refers to the $1 \mathrm{~kb}$ plus Ladder, and $\mathrm{C}$ is control dsRNA without gut content, dsRNA incubated with gut contents for; 30 seconds (lane 1), 1 min (lane 2), 2 min (lane 3), 5 min (lane 4) respectively

mediated biopesticides. This study investigated the RNAi effectiveness in $C$. partellus by targeting four candidate genes (specifically; V-ATPases, Acetylcholinesterase, Chymotrypsin and Arginine kinase) involved in the various biological functions of $C$. partellus. V-ATPase is multisubunit proton pumps that energize transport across plasma membranes in insect cells and epithelia. Acetylcholinesterase is an essential enzyme in the insect central nervous system, which terminates nerve impulse transmission at synaptic junctions of cholinergic neurons through neurotransmitter acetylcholine hydrolyzation. Insects usually exhibit high Acetylcholinesterase expression for accurate nerve impulse termination, a minor decrease in the enzyme activity disrupt nerve impulse transmission and affect insect survival. Chymotrypsin belongs to the serine proteases family involved in various biological functions, 


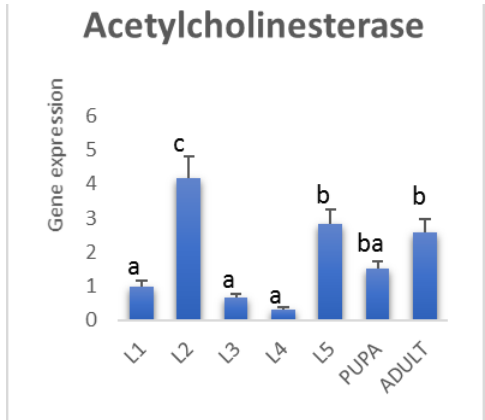

A

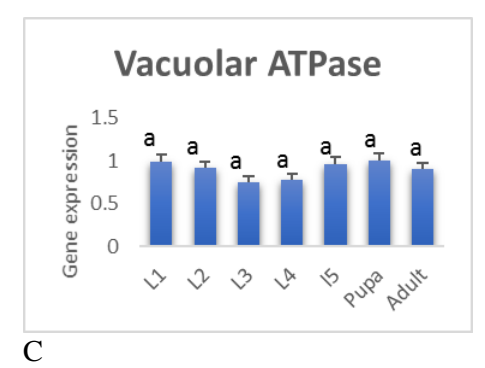

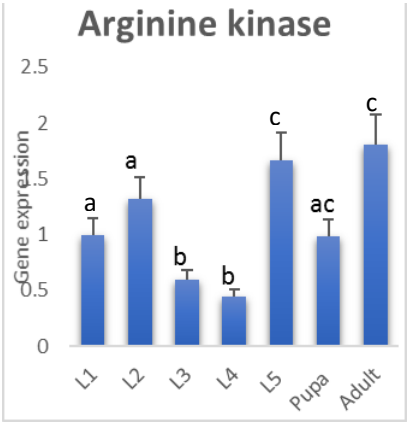

B

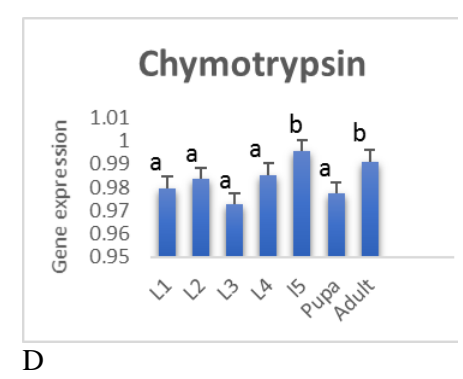

Fig. 9: Temporal expression profiles of the four genes being studied in Chilo partellus. A) acetylcholinesterase expression across all developmental stages, B) arginine kinase expression across all developmental stages, C) vacuolar ATPase expression across all developmental stages, and D) chymotrypsin expression across all developmental stages. Values are expression mean \pm standard error, and different letter indicate significant different $(P<0.05)$
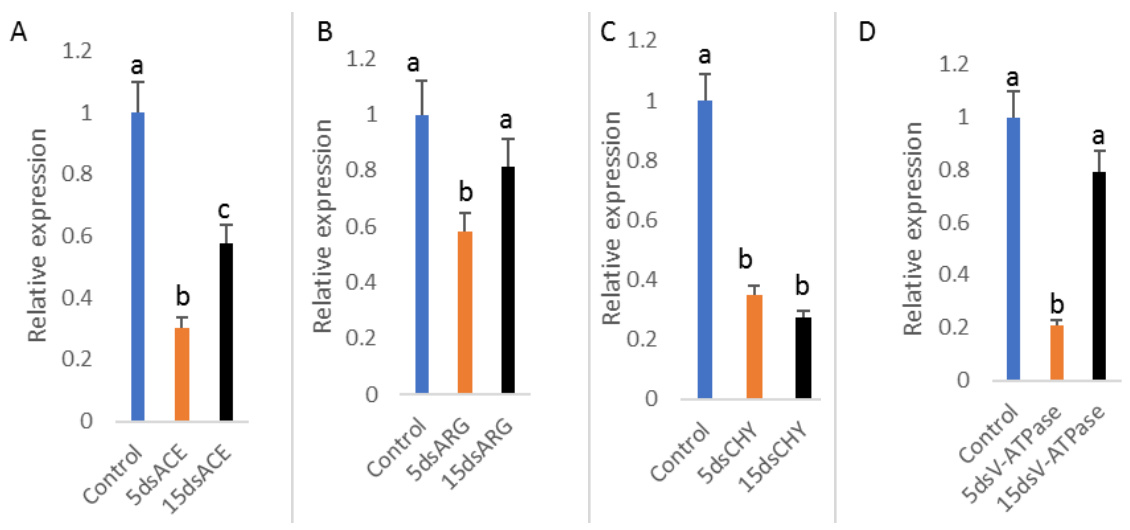

Fig. 10: Relative knockdown in transcript levels of targeted genes in in-vitro feeding assay with bacterially expressed dsRNA for a period of 5 and 15 days. A) Acetylcholinesterase, B) Arginine kinase, C) Chymotrypsin, D) V-ATPase. Orange coloured bar refers to exposure for 5days while the Black coloured bar represents 15 days exposure. Values are expression mean \pm standard error and different letter indicate significant differences $(P<0.05)$ between the two genes and control (without dsRNA treatment

including food digestion, immune defense, and zymogen activation. Arginine kinase $(\mathrm{AK})$ is a phosphotransferase involved in cellular energy metabolism; it catalyzes the transfer of a high-energy phosphate group from ATP to Larginine to produce phospho arginine (Bragg et al. 2012). Different dsRNA delivery methods such as oral feeding, microinjection, soaking, transfection and host plant delivery have evolved over the years. Oral delivery of dsRNA is a proven delivery method of inducing RNAi in Lepidoptera (Choi and Meer 2019). We used an oral route to deliver bacterially-expressed dsRNA and purified dsRNA into the insect gut microenvironment to examine the knockdown efficiency of the targeted genes in C. partellus.

Another critical element in determining the RNAi efficiency is the rate of degradation of dsRNA by haemolymph and gut extracellular ribonucleases ( Wang et al. 2016; Song et al. 2017; Spit et al. 2017). It has been well established that for fruitful RNAi induction, the dsRNA must persist in being absorbed into the insect cell without degradation. We investigated our purified dsRNA under various conditions; to check if the insectary condition would influence dsRNA, we found out that the dsRNA remained 

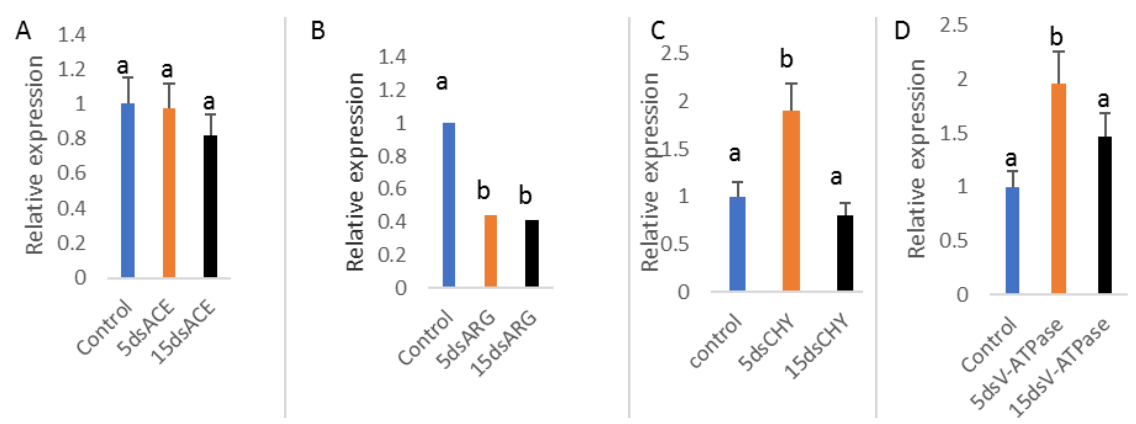

Fig. 11: Relative knockdown in transcript levels of different targeted genes after feeding larvae with purified dsRNA for 5 days and 15 days. A) Acetylcholinesterase B) Arginine kinase, C), Chymotrypsin, D) V-ATPase. Orange coloured bar refers to exposure for 5 days, while the black coloured bar represents 15 days of exposure. Values are expression mean \pm standard error, and the different letter indicates a significant difference $(P<0.05)$ between the two genes and control (without dsRNA treatment)

stable for approximately $72 \mathrm{~h}$. However, the dsRNA was found intact on an artificial diet for about $36 \mathrm{~h}$ and start degrading after $48 \mathrm{~h}$ of exposure. These findings were similar to initial reports documenting that it takes $48 \mathrm{~h}$ to $84 \mathrm{~h}$ for nucleases present in an artificial diet to degrade dsRNA (Christiaens et al. 2014; Cao et al. 2018). Additionally, we found that the $C$. partellus haemolymph and gut content degrade dsRNA at a very rapid rate. The $C$. partellus haemolymph starts dsRNA degradation at about 1 min with complete degradation at $30 \mathrm{~min}$. The instability of dsRNA in gut content and haemolymph limits the RNAi efficiency in C. partellus. This finding is similar to Cooper et al. (2020), who measured dsRNA stability with RT-qPCR and reported high dsRNA degradation in Ostrinia nubilalis's gut content and heamolymph. The rapid gut content degradation of dsRNA in this study is in accordance with previous studies that reported a correlation between dsRNA degradation and low RNAi efficiency in insects (Cooper $e t$ al. 2021; Peng et al. 2018).

Several earlier studies have established that feedingbased RNAi can precisely induce an RNAi response in agricultural insects (Miller et al. 2012; Upadhyay et al. 2013; Abdel-latief and Hoffmann 2014; Xiao et al. 2015; Cappelle et al. 2016). RNAi effectiveness generally depends on the sufficient concentration of ds/siRNA able to initiate the RNAi pathway. However higher concentration is most often used while executing oral feeding assays. We found out that prolonged exposure of $C$. partellus larvae to dsRNA does not lead to enhanced silencing in most targeted genes but rather attempt to nullify the suppression effects. We hypothesize that an alternative pathway might be activated since the mechanisms for this is not clear; further experiments are needed to understand the multiple mechanisms that might contribute to low RNAi efficiency in C. partellus. Our suggestion is that siRNA and shRNA targeting a specific gene may induce nonspecific effects in the stress response pathways (Olejniczak et al. 2010), which may rescue the targeted gene transcript reduction.

The comparison between the transcript knockdowns in larvae fed on bacterially-expressed dsRNA and purified
dsRNAs indicated a significant knockdown of all the four genes evaluated in this study. However, larvae fed on purified dsRNAs failed to induce significant knockdown in most of the targeted genes. The low sensitivity of purified dsRNA can be ascribed to its degradation in the gut due to the presence of nuclease. The knockdown observed in this study indicated that RNAi efficiency varied between target genes in C. partellus. Recently, studies have shown that REase competes with Dicer-2 for targeted dsRNA, influences the unique total reads of target gene siRNAs, and consequently affects RNAi efficiency (Guan et al. 2018). Although different physiological conditions in other tissues modulate enzyme activity and various insects produce a variety of dsRNA degrading enzymes in different quantities (Peng et al. 2018; Cooper et al. 2021).

These results further strengthen the notion that the rapid degradation of dsRNAs affects the ability to induce RNAi mechanism and influence its stability. Since most bacterially-expressed double-stranded dsRNAs exhibited significant knockdown compared to purified dsRNAs. The challenges of the instability of dsRNA and rapid degradation of dsRNA must be overcome to enhance RNAi efficiency in C. partellus. The protection of dsRNA from nuclease will be a better way of achieving optimum knockdown in $C$. partellus and facilitating RNAi-mediated control strategy. Efforts to protect dsRNA from nucleases degradation by silencing nuclease have been demonstrated to enhance dsRNA uptake in agricultural pests and subsequently improve RNAi efficiency (Giesbrecht et al. 2020; Wang et al. 2020; Sharma et al. 2021). Research evidence has shown that nanoparticle-mediated delivery overcomes poor cellular internalization and nucleolytic degradation of dsRNAs for effective RNAi response in insect cells (Christiaens et al. 2018; Dhandapani et al. 2019; Wang et al. 2019; Baddar et al. 2020; Yan et al. 2021).

\section{Conclusion}

The data presented in this study establish that oral feeding of bacterially expressed dsRNA through an artificial diet 
effectively induces RNAi-mediated knockdown of the targeted genes. In contrast, purified dsRNA rarely initiates the RNAi mechanism in $C$. partellus. We suggest that the dsRNA should be protected from direct access with nucleases for optimum RNAi in $C$. partellus. Nanoparticles are currently being employed as novel delivery vehicles that enhance dsRNA uptake into insect cells and protect dsRNA against RNases. Future nanoparticle-mediated delivery of the dsRNAs examined in this study is worth investigation to evaluate the best dsRNA delivery approaches in C. partellus.

\section{Acknowledgements}

We acknowledge The World Academy of Sciences for awarding the TWAS-CEMB Postgraduate Fellowship and International Foundation for Science for providing the grant for this research.

\section{Author Contributions}

Olawale Samuel Adeyinka performed the experiment and write the manuscript, Bushra Tabassum and Idrees Ahmad Nasir designed and supervised the experiment and edit the manuscript. Nida Toufiq, Iqra Yousaf and Abimbola Pius Okiki reviewed the manuscript and effect corrections and Samam Riaz executed the insect bioassay and real time assays.

\section{Conflict of Interest}

All the authors declare no conflicts of interest

\section{Data Availability}

All the data used to support the findings of this study are included in the article.

\section{Ethics Approval}

Not applicable.

\section{Funding Source}

This study was supported by International Foundation for Science grant (No. C/6194-1).

\section{Reference}

Abbasi R, D Heschuk, B Kim, S Whyard (2020). A novel paperclip doublestranded RNA structure demonstrates clathrin-independent uptake in the mosquito Aedes aegypti. Ins Biochem Mol Biol 127:103492

Abdel-latief M, KH Hoffmann (2014). Peptides Functional activity of allatotropin and allatostatin in the pupal stage of a holometablous insect, Tribolium castaneum. Peptides 53:172-184

Adeyinka OS, S Riaz, N Toufiq, I Yousaf, MU Bhatti, A Batcho, AA Olajide, IA Nasir, B Tabassum (2020). Advances in exogenous RNA delivery techniques for RNAi-mediated pest control. Mol Biol Rep 47:6309-6319
Adeyinka OS, B Tabassum, IA Nasir, I Yousaf, IA Sajid, K Shehzad, A Batcho, T Husnain (2019). Identification and validation of potential reference gene for effective dsRNA knockdown analysis in Chilo partellus. Sci Rep 9; Article 13629

Adeyinka OS, T Bushra, NS Muhammad, MU Bhatti, AN Idrees, H Tayyab (2018). A lag in the advancement of biotechnology: Reliable control of maize stem borers in Africa. J Plant Prot Res 54:8-24

Ajala SO, AM Nour, MO Odindo (2010). Evaluation of maize (Zea mays L.) genotypes as a component of integrated stem borer (Chilo partellus Swinhoe) management in coastal region of Kenya. J Agric Res 5:758-763

Bragg J, A Rajkovic, C Anderson, R Curtis, J Van Houten, B Begres, C Naples, M Snider, D Fraga, M Singer (2012). Identification and characterization of a putative arginine kinase homolog from Myxococcus xanthus required for fruiting body formation and cell differentiation. J Bacteriol 194:2668-2676

Camargo RA, GO Barbosa, IP Possignolo, LEP Peres, E Lam, JE Lima, A Figueira, H Marques-Souza (2016). RNA interference as a gene silencing tool to control Tuta absoluta in tomato (Solanum lycopersicum). PeerJ 4; Article e2673

Cao M, JA Gatehouse, EC Fitches (2018). A systematic study of RNAi effects and dsRNA stability in Tribolium castaneum and Acyrthosiphon pisum, following injection and ingestion of analogous dsRNAs. Intl J Mol Sci 19:1079-1096

Cappelle K, CFRD Oliveira, BV Eynde, O Christiaens, G Smagghe (2016). The involvement of clathrin-mediated endocytosis and two Sid-1like transmembrane proteins in double-stranded RNA uptake in the Colorado potato beetle midgut. Ins Mol Biol 25:315-323

Choi MY, RKV Meer (2019). Phenotypic Effects of PBAN RNAi Using Oral Delivery of dsRNA to Corn Earworm (Lepidoptera: Noctuidae) and Tobacco Budworm Larvae. J Econ Entomol 112:434-439

Christiaens O, MG Tardajos, ZLM Reyna, M Dash, P Dubruel, G Smagghe (2018). Increased RNAi Efficacy in Spodoptera exigua via the formulation of dsRNA with guanylated polymers. Front Physiol 9; Article 316

Christiaens O, L Swevers, G Smagghe, L Sweveres, G Smagghe (2014). DsRNA degradation in the pea aphid (Acyrthosiphon pisum) associated with lack of response in RNAi feeding and injection assay. Peptides 53:307-314

Colmenares SU, SM Buker, M Bühler, M Dlakic, D Moazed (2007) Coupling of double-stranded RNA synthesis and siRNA generation in fission yeast RNAi. Mol Cell 27:449-461

Cooper AMW, H Song, Z Yu, M Biondi, J Bai, X Shi, Z Ren, SM Weerasekara, DH Hua, K Silver, J Zhang, KY Zhu (2021). Comparison of strategies for enhancing interference efficiency in Ostrinia nubilalis. Pest Manage Sci 77:635-645

Cooper AMU, Z Yu, M Biondi, H Song, K Silver, J Zhang, KY Zhu (2020). Stability of double-stranded RNA in gut contents and hemolymph of Ostrinia nubilalis larvae. Pest Biochem Physiol 169:104672

Dhandapani RK, D Gurusamy, JL Howell, SR Palli (2019). Development of CS-TPP-dsRNA nanoparticles to enhance RNAi efficiency in the yellow fever mosquito, Aedes aegypti. Sci Rep 9; Article 8775

Dias NP, D Cagliari, EAD Santos, G Smagghe, JL Jurat-Fuentes, S Mishra, DE Nava, MJ Zotti (2020). Insecticidal Gene Silencing by RNAi in the Neotropical Region. Neotrop Entomol 49:1-11

Elbashir SM (2001). Functional anatomy of siRNAs for mediating efficient RNAi in Drosophila melanogaster embryo lysate. EMBO J 20:68776888

Elhaj Baddar Z, D Gurusamy, J Laisney, P Tripathi, SR Palli, JM Unrine (2020). Polymer-coated hydroxyapatite nanocarrier for doublestranded RNA delivery. J Agric Food Chem 68:6811-6818

Fire A, S Xu, MK Montgomery, SA Kostas, SE Driver (1998). Potent and specific genetic interference by double-stranded RNA in Caenorhabditis elegans. Nature 391:806-811

Ghosh SKB, WB Hunter, AL Park, DE Gundersen-Rindal (2017). Double strand RNA delivery system for plant-sap-feeding insects. PLoS One 12; $\mathrm{e} 0171861$

GiesbrechtD,DHeschuk, IWiens, D Boguski, P LaChance, S Whyard (2020). RNA interference is enhanced by knockdown of double-stranded RNases in the yellow fever mosquito Aedes aegypti. Ins 11; Article 327 
Guan RB, HC Li, YJ Fan, SR Hu, O Christiaens, G Smagghe, XX Miao (2018). A nuclease specific to lepidopteran insects suppresses RNAi. J Biol Chem 293:6011-6021

Husain M, KG Rasool, M Tufail, WS Alwaneen, AS Aldawood (2021). RNAi-mediated silencing of vitellogenin gene curtails oogenesis in the almond moth Cadra cautella. PLoS One 16; Article e0245928

Jain RG, KE Robinson, SAsgari, N Mitter (2021). Current scenario of based hemipteran control. Pest Manage Sci 77:2188-2196

Khadioli N, ZEH Tonnang, E Muchugu, G Ong'amo, T Achia, I Kipchirchir, J Kroschel, BL Ru (2014). Effect of temperature on the phenology of Chilo partellus (Swinhoe) (Lepidoptera, Crambidae); simulation and visualization of the potential future distribution of $C$. partellus in Africa under warmer temperatures through the development of life-table param. Bull Entomol Res 104:809-22

Li X, X Dong, C Zou, H Zhang (2015). Endocytic pathway mediates refractoriness of insect Bactrocera dorsalis to RNA interference. Sci Reports 5; Article 8700

Livak KJ, TD Schmittgen (2001). Analysis of relative gene expression data using real-time quantitative PCR and the $2-\Delta \Delta \mathrm{CT}$ Method. Methods 25:402-408

Lü J, Z Liu, W Guo, M Guo, S Chen, H Li, C Yang, Y Zhang, H Pan (2019). Feeding delivery of dsHvSnf7 is a promising method for management of the Pest Henosepilachna vigintioctopunctata (Coleoptera: Coccinellidae). Insects 11; Article 34

Mello CC, DJ Conte (2004). Revealing the world of RNA interference. Nature 431:338-342

Miller SC, K Miyata, SJ Brown, Y Tomoyasu (2012). Dissecting systemic RNA interference in the red flour beetle Tribolium castaneum: Parameters affecting the efficiency of RNAi. PLoS One 7; Article e47431

Miyata K, P Ramaseshadri, Y Zhang, G Segers, R Bolognesi, Y Tomoyasu (2014). Establishing an in vivo assay system to identify components involved in environmental RNA interference in the western corn rootworm. PLoS One 9; Article e101661

Mugo S, J Songa, H Degroote, D Hoisington (2002). Insect resistant maize for Africa (IRMA) project: An overview. In: Syngenta Symposium Proceedings, Vol. 25, pp:1-16, Washington DC, USA.

Olejniczak M, P Galka, WJ Krzyzosiak (2010). Sequence-non-specific effects of RNA interference triggers and microRNA regulators. $\mathrm{Nucl}$ Acids Res 38:1-16

Park J, K Lee, S Lee, W Oh, P Jeong, T Woo, C Kim, Y Paik, H Koo (2008). The efficiency of RNA interference in Bursaphelenchus xylophilus. Mol Cells 26:81-86

Peng Y, K Wang, W Fu, C Sheng, Z Han (2018). Biochemical comparison of dsRNA degrading nucleases in four different insects. Front Physiol 9; Article 24

Pinheiro DH, AM Vélez, E Fishilevich, H Wang, NP Carneiro, A ValenciaJiménez, FH Valicente, KE Narva, BD Siegfried (2018). Clathrindependent endocytosis is associated with RNAi response in the western corn rootworm, Diabrotica virgifera virgifera LeConte. PLoS One 13; Article e0201849

Pratt CF, KL Constantine, ST Murphy (2017). Economic impacts of invasive alien species on African smallholder livelihoods. Glob Food Secur 14:31-37
Schwarz D, G Hutvágner, T Du, Z Xu, N Aronin, P Zamore (2003). Complex, Asymmetry in the assembly of the RNAi enzyme. Cell 115:199-208

Sharma R, CNT Taning, G Smagghe, O Christiaens (2021). Silencing of double-stranded ribonuclease improves oral RNAi efficacy in southern green stinkbug Nezara viridula. Insects 12:115-130

Song H, J Zhang, D Li, AMW Cooper, K Silver, T Li, X Liu, E Ma, KY Zhu, J Zhang (2017). A double-stranded RNA degrading enzyme reduces the efficiency of oral RNA interference in migratory locust. Ins Biochem Mol Biol 86:68-80

Spit J, A Philips, N Wynant, D Santos, G Plaetinck, J Vanden Broeck (2017). Knockdown of nuclease activity in the gut enhances RNAi efficiency in the colorado potato beetle, Leptinotarsa dece mLineata, but not in the desert locust, Schistocerca gregaria. Ins Biochem Mole Biol 81:103-116

Tamiru A, E Getu, B Jembere, T Bruce (2012). Effect of temperature and relative humidity on the development and fecundity of Chilo partellus (Swinhoe) (Lepidoptera: Crambidae). Bull Entomol Res 102:9-15

TimAR, K Ginalski, NV Grishin, X Wang (2004). Biochemical identification of Argonaute 2 as the sole protein required for RNA-induced silencing complex activity. Proc Natl Acad Sci 101:14385-14389

Upadhyay SK, S Dixit, S Sharma, H Singh, J Kumar (2013). siRNA machinery in whitefly (Bemisia tabaci). PLoS One 8; Article e83692

Verger PJP, AR Boobis (2013). Reevaluate pesticides for food security and safety. Science 341:717-718

Vogel E, D Santos, L Mingels, TW Verdonckt, JV Broeck (2019). RNA interference in insects: Protecting beneficials and controlling pests. Front Physiol 9; Article 1912

Wang K, Y Peng, J Chen, Y Peng, X Wang, Z Shen, Z Han (2020). Comparison of efficacy of RNAi mediated by various nanoparticles in the rice striped stem borer (Chilo suppressalis). Pest Biochem Physiol 165:104467

Wang K, Y Peng, J Pu, W Fu, J Wang, Z Han (2016). Variation in RNA efficacy among insect species is attributable to dsRNA degradation in vivo. Ins Biochem Mol Biol 77:1-9

Wang W, Q Pan, B Tian, F He, Y Chen, G Bai, A Akhunova, HN Trick, E Akhunov (2019). Gene editing of the wheat homologs of TONNEAU 1-recruiting motif encoding gene affects grain shape and weight in wheat. Plant J 100:251-264

Worrall EA, A Bravo-Cazar, AT Nilon, SJ Fletcher, KE Robinson, JP Carr, N Mitter (2019). Exogenous application of RNAi-inducing doublestranded RNA inhibits aphid-mediated transmission of a plant virus. Front Plant Sci 10; Article 265

Xiao D, X Gao, J Xu, X Liang, Q Li, J Yao, K Zhu (2015). Clathrindependent endocytosis plays a predominant role in cellular uptake of double-stranded RNA in the red flour beetle. Ins Mol Biol 60:68-77

Yan S, B Ren, J Shen (2021). Nanoparticle-mediated double-stranded RNA delivery system: A promising approach for sustainable pest management. Ins Sci 28:21-34

Ye X, L Yang, D Stanley, F Li, Q Fang (2017). Two Bombyx mori acetylcholinesterase genes influence motor control and developmen in different ways. Sci Rep 7; Article 4985-

Zhu KY, SR Palli (2020). Mechanisms, applications, and challenges of insect RNA interference. Anпи Rev Entomol 65:293-311 